\title{
LINEAR POLARIZATION OF A DOUBLE PEAKED BROAD EMISSION LINE IN ACTIVE GALACTIC NUCLEI
}

\author{
HeE-Won LeE \\ Department of Astronomy and Space Science, Sejong University, Seoul 143-747, Korea \\ E-mail : hwlee@sejong.ac.kr \\ (Received February 18, 2011; Revised March 28, 2011; Accepted March 28, 2011)
}

\begin{abstract}
A small number of active galactic nuclei are known to exhibit prominent double peak emission profiles that are well-fitted by a relativistic accretion disk model. We develop a Monte Carlo code to compute the linear polarization of a double peaked broad emission line arising from Thomson scattering. A Keplerian accretion disk is adopted for the double peak emission line region and the geometry is assumed to be Schwarzschild. Far from the accretion disk where flat Minkowski geometry is appropriate, we place an azimuthally symmetric scattering region in the shape of a spherical shell sliced with $\Delta \mu=0.1$. Adopting a Monte Carlo method we generate line photons in the accretion disk in arbitrary directions in the local rest frame and follow the null geodesic paths of the photons until they hit the scattering region. The profile of the polarized flux is mainly determined by the relative location of the scattering region with respect to the emission source. When the scattering region is in the polar direction, the degree of linear polarization also shows a double peak structure. Under favorable conditions we show that up to $0.6 \%$ linear polarization may be obtained. We conclude that spectropolarimetry can be a powerful probe to reveal much information regarding the accretion disk geometry of these active galactic nuclei.
\end{abstract}

Key words : galaxies: active — galaxies: nuclei — quasars: emission lines — radiative transfer polarization

\section{INTRODUCTION}

Active galactic nuclei (hereafter AGN) are characterized by nonthermal continuum with prominent emission lines. It is believed that the central engines of AGN consist of a supermassive black hole surrounded by an accretion disk. AGN emission lines are usually classified by the width, where forbidden lines including $[\mathrm{O}$ III] $\lambda 5007$ exhibit narrow width of about $500 \mathrm{~km} \mathrm{~s}^{-1}$. In contrast to forbidden lines, permitted lines exhibit very large widths typically an order of magnitude larger than those of forbidden lines (e.g., Osterbrock \& Ferland 2005).

The AGN emission lines are believed to be Dopplerbroadened, and therefore broad lines are particularly important kinematic diagnostics of the central regions of AGN. The reverberation mapping method may be used to locate the broad permitted emission line region within a fraction of light month from the central engine (Blandford \& McKee 1982). The profiles of most broad emission lines show a single broad peak and may be fitted by multiple Gaussian functions (Laor et al. 1994). It appears that the broad emission region is composed of a large number of small optically thick clouds moving very fast near the central massive black hole (Arav et al. 1998).

Despite intensive efforts, there has been a lack of direct observational evidence indicating the presence of an accretion disk in the central region of AGN. One ar- gument for the presence of the accretion disk is the big blue bump in AGN spectra. It was also proposed that a partial obscuration by an accretion disk may leave an absorption edge near the Lyman limit (Koratkar et al. 1995).

A small number of AGN exhibit double peaked broad emission lines in their spectra (Eracleous \& Halpern 1994). These AGNs were discovered in broad line radio galaxies. Later, from the Sloan Digital Sky Survey project, double peak emitters were additionally found among radio-quiet AGNs (Puchnarewicz et al. 1996). Eracleous et al. (1995) summarized the observational properties of these double peak emission AGNs as follows: (a) Balmer lines are on the average twice as broad as those of typical AGNs, (b) in the optical spectrum starlight fraction is considerably larger than typical AGNs, (c) low ionization forbidden lines have large equivalent width and large $[\mathrm{O} \mathrm{I}] /[\mathrm{O} \mathrm{III}]$ flux ratio compared to those of typical AGNs.

In these AGNs discovered earlier, the blue peak is conspicuously stronger than the red one. Chen et al. (1989) computed line profiles assuming that the emission region is in the form of a finite disk rotating with a Keplerian velocity around a Schwarzschild black hole. In this case the stronger blue peak is a natural consequence of the relativistic Doppler boosting. Their profile fitting is quite successful considering the small number of fitting parameters such as the inner and outer 
radii of the emission disk, the inclination angle and emissivity given in the form of a power law of the radius. An improved profile fitting was provided by Chen \& Halpern (1989).

The situation became more complicated when double peak emitters with stronger red peak were reported. Eracleous et al. (1995) invoked an elliptical disk model to explain these line profiles. The accretion disk emission model is not the unique successful model for the double peaked profiles. For example, binary broad line regions were proposed by Gaskell (1996), and double sided jets were considered by Zheng et al. (1990).

Spectropolarimetry contributed greatly to the unification model of AGNs (e.g., Antonucci 1993). Broad polarized flux of Balmer lines in the prototypical Seyfert 2 galaxy NGC 1068 reveals the broad emission line region that is obscured from the line of sight in the case of type 2 AGNs (Antonucci \& Miller 1985). The constant position angle and degree of polarization may indicate the fact that Thomson scattering is responsible for the polarized flux. Laor (2006) investigated the $\mathrm{H} \alpha$ emission line in NGC 4395 which shows quite extended wings $\geq 2500 \mathrm{~km} \mathrm{~s}^{-1}$. He proposed electron scattering in the broad line region as the mechanism for the formation of wings and thereby deduced a Thomson scattering optical depth of $\tau_{e}=0.34$.

Spectropolarimetry provides views of two different line of sights for the accretion disk emission region, so that more refined models may be established (e.g., Lee \& Blandford 1997). In this paper, we compute the linearly polarized flux by adopting an accretion disk model where a free electron region is additionally introduced.

\section{MODEL AND CALCULATION}

\subsection{Keplerian Circular Motion in a Schwarz- schild Geometry}

Geodesics in Schwarzschild metric are detailed in many textbooks of general relativity (e.g., Misner, Thorne \& Wheeler 1973). Luminet (1979), in particular, described the structure of null geodesics of the Schwarzschild metric in detail in order to construct various images of the accretion disk around the black hole. In this subsection, we describe the line profile from a Keplerian disk around a Schwarzschld black hole with the mass $M$.

For simplicity, we use geometrized units by setting $c=G=1$. The metric adopted in this work is

$$
d s^{2}=-\left(1-\frac{2 M}{r}\right) d t^{2}+\frac{d r^{2}}{1-(2 M / r)}+r^{2} d \Omega^{2}
$$

where the angular part is defined by $d \Omega^{2}=d \theta^{2}+$ $r \sin ^{2} \theta d \phi^{2}$.

The emitting particle is in Keplerian motion in the direction $\overrightarrow{\mathbf{e}}_{\phi}$ with $\theta=\pi / 2$, for which the Lagrangian function $L$ is

$$
L=-\left(1-\frac{2 M}{r}\right) \dot{t}^{2}+\frac{\dot{r}^{2}}{1-(2 M / r)}+r^{2} \dot{\phi}^{2} .
$$

Here, the dot represents the derivative with respect to the proper time $\tau$. From this Lagrangian, we have an equation of motion in a form of energy conservation

$$
\tilde{E}_{1}^{2}=\dot{r}^{2}+\left(1-\frac{2 M}{r}\right)\left(1+\frac{\tilde{l}_{1}^{2}}{r^{2}}\right),
$$

where $\tilde{E}_{1}=\dot{t}(1-2 M / r)$ and $\tilde{l}_{1}=r^{2} \dot{\phi}$ are the energy and angular momentum integrals of the emitting particle. For a Keplerian circular motion, we have $\dot{r}=0$ and the effective potential has a minimum there. Therefore, if we introduce the effective potential $V_{e f f}$ as a function of $u=1 / r$ defined by

$$
V_{e f f}(u)=(1-2 M u)\left(1+\tilde{l}_{1}^{2} u^{2}\right),
$$

then the circular motion occurs at the minimum of $V_{\text {eff }}$. We also have

$$
\tilde{l}_{1}^{2}=\frac{M r^{2}}{r-3 M}, \quad \tilde{E}_{1}^{2}=\frac{(r-2 M)^{2}}{r(r-3 M)} .
$$

Substituting Eq. (5) into Eq. (2), we obtain the coordinate velocities

$$
\dot{t}=\frac{d t}{d \tau}=\sqrt{\frac{r}{r-3 M}}, \quad \dot{\phi}=\sqrt{\frac{M}{r^{2}(r-3 M)}} .
$$

The four-velocity of the emitting particle in Kepelerian circular motion is given by

$$
u^{\alpha}=[\dot{t}, \dot{r}, \dot{\theta}, \dot{\phi}]=\dot{t}\left[1,0,0, \sqrt{M / r^{3}}\right],
$$

which leads to the same form as the classical Keplerian angular speed $d \phi / d t=(\dot{\phi} / \dot{t}) \propto r^{-3 / 2}$ (e.g., Shapiro \& Teukolsky 1986).

\subsection{Null Geodesics in Schwarzschild Metric}

Due to spherical symmetry of the Schwarzschild geometry, all geodesics lie in a plane. We first follow the formalism introduced by Luminet (1979) for photon paths in the equatorial plane, which, in turn, are transformed into those in an arbitrarily oriented plane.

We assume that a photon initially at a position $(t, r, \theta=\pi / 2, \phi)$ in a Keplerian disk is emitted in the direction $\hat{\mathbf{k}}$ making an angle $\alpha$ with respect to the radial direction $\overrightarrow{\mathbf{e}}_{r}$ as measured by the local observer. Here, $\hat{\mathbf{k}}$ is considered as a 3 -spatial directional vector. First, we describe the trajectory in the equatorial plane and then we transform into a plane with an arbitrary angle $\beta$ with respect to the equatorial plane. 
For a photon traveling on the equatorial plane, the impact parameter $b$ is given by

$$
b=\frac{r \sin \alpha}{\sqrt{1-(2 M / r)}},
$$

which is related to the periastron distance $P$ by the relation

$$
b^{2}=\frac{P^{3}}{P-2 M} .
$$

Therefore, given $b$, a cubic equation for $P$ is written as

$$
P^{3}-b^{2} P+2 M b^{2}=0 .
$$

Defining $b_{\text {crit }}=3 \sqrt{3} M$, we see that the cubic equation has no positive root for $b<b_{c r i t}$, for which the photon paths are characterized by vanishing periastron distance. This implies that the null geodesic may extend to the singularity of the Schwarzschild geometry.

When $b>b_{\text {crit }}$, the cubic equation has three real roots. This condition is equivalent to the requirement

$$
\sin \alpha>\frac{b_{c r i t} \sqrt{1-(2 M / r)}}{r}
$$

which describes the angular range of the sky seen from a local observer located at $r$ (see e.g., Shapiro \& Teukolsky 1986). Among the 3 real roots for $b>3 \sqrt{3} M$, only one root is larger than $b / \sqrt{3}$, which is physically meaningful, and is explicitly written as

$$
P=\frac{2 b}{\sqrt{3}} \cos \left(\frac{\pi}{3}-\frac{1}{3} \tan ^{-1} \sqrt{\frac{b^{2}}{b_{c r i t}^{2}}-1}\right) .
$$

As is shown by Luminet (1979), the angular distance traversed by a photon from the initial position to the asymptotic observer is given by

$$
\Delta \alpha=2\left(\frac{P}{Q}\right)^{1 / 2}\left[F\left(\zeta_{r}, k\right)-F\left(\zeta_{\infty}, k\right)\right] .
$$

Here, $F(\zeta, k)=\int_{0}^{\zeta}\left(1-k^{2} \sin ^{2} x\right)^{-1 / 2} d x$ is the elliptic integral of modulus $k$ and the parameter $Q$ is given by

$$
Q=(P-2 M) /(P+6 M) .
$$

Other parameters are defined as

$$
\begin{aligned}
k^{2} & =(Q-P+6 M) /(2 Q), \\
\sin ^{2} \zeta_{r} & =[Q-P+2 M+(4 M P / r)] /(Q-P+6 M), \\
\sin ^{2} \zeta_{\infty} & =(Q-P+2 M) /(Q-P+6 M) .
\end{aligned}
$$

Given the initial position and wavevector $\hat{\mathbf{k}}$ of the photon, these parameters are calculated in a straightforward manner to yield the final wavevector $\hat{\mathbf{k}}_{f}$

$$
\hat{\mathbf{k}}_{f}=\cos \alpha_{f} \overrightarrow{\mathbf{e}}_{\hat{r}}+\sin \alpha_{f} \overrightarrow{\mathbf{e}}_{\hat{\phi}},
$$

which is detected by an observer far from the black hole Here, $\alpha_{f}=\alpha+\Delta \alpha$.

Now we transform the final photon wavevector $\hat{\mathbf{k}}_{f}$ lying in the equatorial plane into the wavevector $\hat{\mathbf{k}}_{o}$ in the plane with an arbitrary angle $\beta$ with respect to the equatorial plane. In terms of the angle $\beta, \hat{\mathbf{k}}_{o}$ is represented as

$$
\hat{\mathbf{k}}_{o}=\cos \alpha_{f} \hat{\mathbf{r}}+\sin \alpha_{f}(-\sin \beta \hat{\theta}+\cos \beta \hat{\phi}) .
$$

Finally, the local energy $h \nu_{e}$ of the photon measured in the rest frame of emitter is related to the observed photon energy $h \nu$ by an observer far from the black hole by the relation

$$
\begin{aligned}
h \nu_{e} & =-p^{\mu} u_{\mu} \\
& =h \nu\left(\frac{1-b M^{1 / 2} r^{-3 / 2} \cos \beta}{\sqrt{1-(3 M / r)}}\right),
\end{aligned}
$$

which was given by Luminet (1979) and was also used by Chen et al. (1989). Here, use is made of the fact that the photon 4 -momentum vector is given by

$$
\begin{aligned}
p^{\mu}= & h \nu\left[(1-2 M / r)^{-1},\left\{1-b^{2}(1-2 M / r) r^{-2}\right\}^{1 / 2},\right. \\
& \left.-b r^{-2} \sin \beta, b r^{-2} \cos \beta\right] .
\end{aligned}
$$

\subsection{Scattering Geometry and Linear Polariza- tion}

We assume that the accretion disk lies in the $x-y$ plane and that the black hole of mass $M$ is located at the center of our coordinate system. We denote by $R_{o}$ and $R_{i}$ the outer and inner radii of the disk. We take the scattering region as a sliced part of a uniform spherical shell with radius much larger than $R_{o}$. We assume that the shell is sufficiently far away from the black hole, so that the scattering region is described by the Minkowski geometry. The uniform shell is sliced by the values of $\mu_{S}=\cos \theta_{S}$ with the bin size of $\Delta \mu_{S}=$ 0.1 , where $\theta$ is the colatitude of the spherical shell. In this work, we consider three different shell segments with $0.75<\mu<0.85,0.45<\mu<0.55$ and $0.15<$ $\mu<0.25$. Fig. 1 shows a schematic illustration of our scattering geometry.

It is also assumed that the scattering region is composed of free electrons, where Thomson scattering gives rise to polarized flux towards an observer in a specified direction. We characterize the scattering region by the Thomson scattering optical depth $\tau_{e}$ in the normal or radial direction. In this work, for simplicity, we consider the two cases where $\tau_{e}=0.4$ and $\tau_{e}=0.8$.

In the Monte Carlo code, we generate a line photon in the accretion disk with an arbitrary direction in the local rest frame. We subsequently compute the additional angular distance traversed by the photon to an asymptotically flat region, for which the mathematical expression is given by Eq. (13). In this work, we assume that the surface emissivity is proportional to $r^{-q}$, 


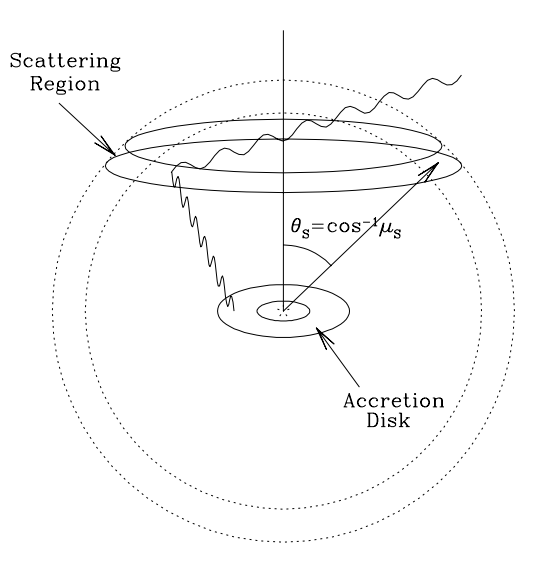

Fig. 1. - Scattering geometry. The scattering region is a sliced uniform shell making an opening angle of $\theta_{S}$ with respect to the center where the supermassive black hole resides. The accretion disk is characterized by inner and outer radii $R_{i}, R_{o}$. The scattering region is also characterized by the Thomson scattering optical depth $\tau_{e}$ in the radial direction. In this work, two cases of $\tau_{e}=0.4$ and $\tau_{e}=0.8$ are considered.

where $q=3.1$ is fixed to the value adopted by Chen et al. (1989). The initial photons are assumed to be completely unpolarized and emitted isotropically.

In the case that the emergent photon enters the scattering region, we obtain a scattered photon using a Monte Carlo technique and compute the degree of polarization. For the description of the Thomson scattering process and polarization, use is made of the density matrix formalism, of which a detailed explanation can be found in the literature (e.g., Lee \& Ahn 1998; Lee \& Blandford 1997).

\section{RESULT}

\subsection{Line Profile and Code Check}

Chen et al. (1989) computed the emission line profiles from a circular Keplerian accretion disk governed by Schwarzschild geometry and showed that the profiles are well fitted to the observed data of Arp 102B. In Fig. 2, as a check of our Monte Carlo code, we reproduce the emission line profiles using the two methods, one being our Monte Carlo code and the other the numerical method provided by Chen et al. (1989). Our Monte Carlo results are illustrated by the crosses and the solid lines show the results obtained using the method of Chen et al. (1989).

The radii of the disk are fixed to be $R_{o}=1000 R_{S c h}$,
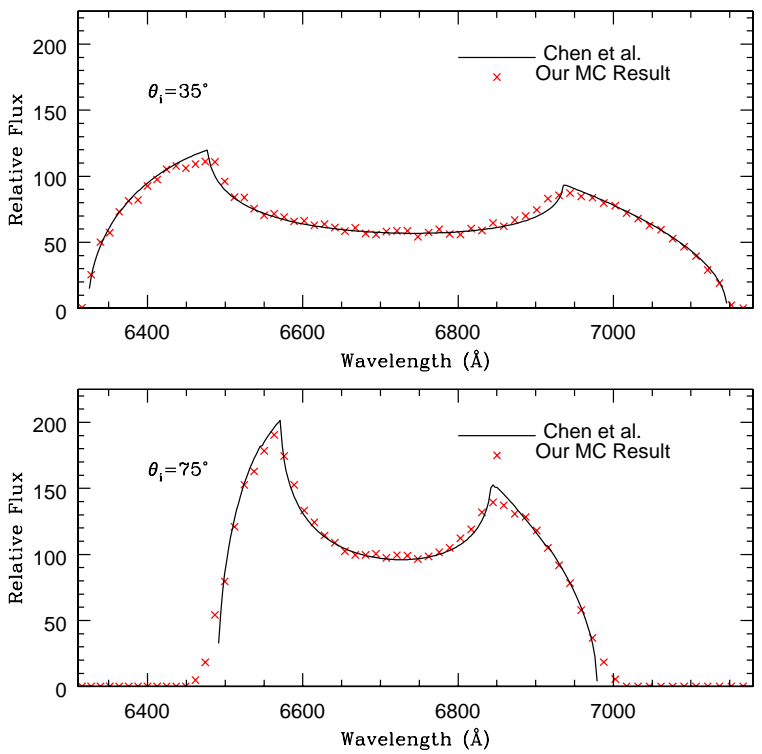

Fig. 2.- Line profiles from a Keplerian disk around a Schwarzschild black hole. The solid lines show the results calculated by the numerically iterative method adopted by Chen et al. (1989) and the crosses show our Monte Carlo results. The observer's viewing angle for the upper panel is $\theta_{i}=35^{\circ}$ and the lower panel is for $\theta_{i}=75^{\circ}$.

$R_{i}=400 R_{S c h}$, where $R_{S c h}=2 G M / c^{2}$ is the Schwarzschild radius. We show two cases of the inclination angle $\theta_{i}$, where the upper panel shows $\theta_{i}=35^{\circ}$ and the lower panel is for $\theta_{i}=75^{\circ}$. The horizontal axis is the observed wavelength assuming that the line emission is $\mathrm{H} \alpha$ and the redshift of the hypothetical galaxy is $z=0$. As is shown in the figure, the agreement between the two methods is excellent except possibly in the neighborhood of the sharp peaks.

\subsection{Degree of Linear Polarization and Polar- ized Flux}

In Fig. 3 we show our Monte Carlo result for $\tau_{e}=0.4$ and the scattering region is a sliced uniform shell with $0.15<\mu_{S}<0.25$. This means that the scattering region lies mostly in the equatorial direction. The top panel shows the line profiles of the total flux for three observer's lines of sight $\mu_{o b}=0.2,0.5$ and 0.8 . In this work, we are interested only in the line shape so that the total flux is normalized arbitrarily.

The bottom panel shows the percentage degree of linear polarization for the same model parameters. The position angle of the linear polarization is shown by the sign of the degree of linear polarization, where the positive degree of polarization represents the polarization in the direction parallel to the disk plane and the negative 


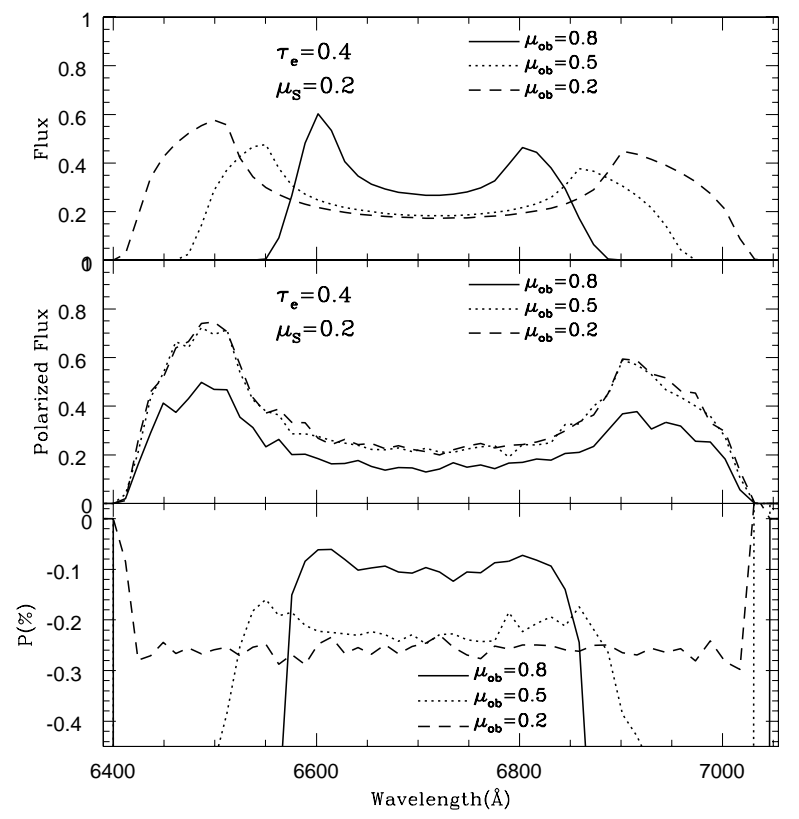

Fig. 3.- Profiles and degree of linear polarization of a double peak emission line in AGN. The scattering region lies in the range $0.15<\mu_{S}<0.25$ and the Thomson scattering optical depth is $\tau_{e}=0.4$. The solid lines are Monte Carlo data for an observer's line of sight with $\mu_{o b}=0.8$, the dotted lines show the result for an observer with $\mu_{o b}=0.5$ and the short dashed lines are for an observer with $\mu_{o b}=0.2$.

degree of polarization in the direction perpendicular to the disk plane.

For observers with $\mu_{o b}=0.2$ and 0.5 , the degree of linear polarization is quite high in the extreme wing parts. However, there is so little photon flux there, which contributes negligibly to the polarized flux. In view of this, we concentrate on the main part of the polarized flux where the degree of linear polarization is nearly constant.

The middle panel shows the linearly polarized flux, which is equal to the product of the total flux and the absolute value of the degree of linear polarization.

The degree of linear polarization is nearly constant in the main part of the total flux. The lowest degree of polarization is obtained for the observer in the polar direction, because of the azimuthal symmetry of the system. The highest degree of linear polarization is obtained for the observer with $\mu_{o b}=0.2$ in the line center. However, the polarized fluxes are similar for observers with $\mu_{o b}=0.2$ and with $\mu_{o b}=0.5$. The overall shapes of the polarized fluxes are similar irrespective of the observer's line of sight and only differ in strength, because they share the same scattering region.

In Fig. 4 we show the Monte Carlo result for a scattering region lying in the polar direction with

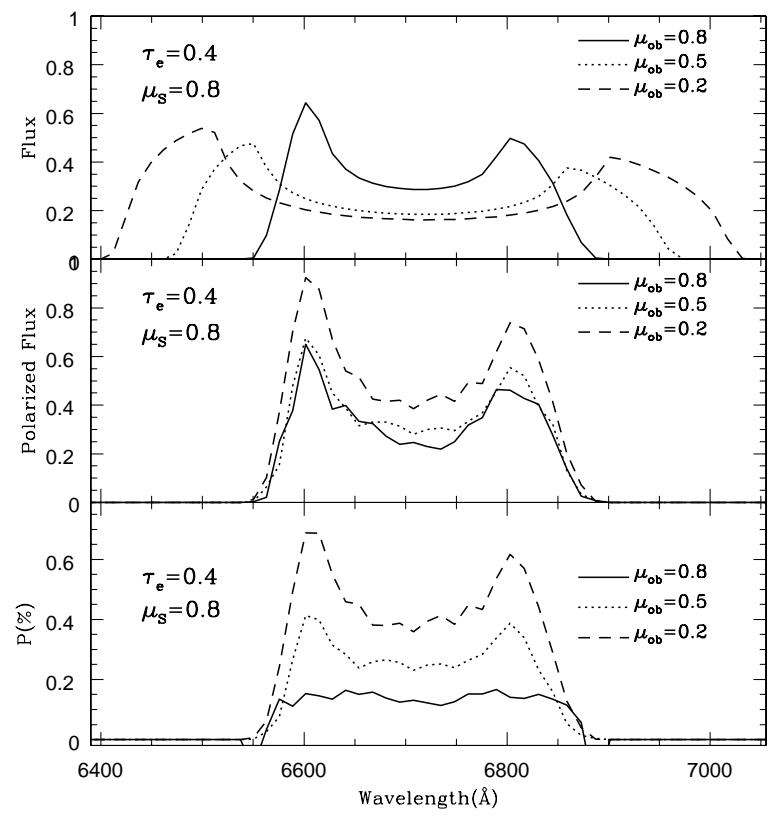

Fig. 4. - Same as Fig. 3 except that the scattering region lines in the polar direction with $0.75<\mu_{S}<0.85$. Note that the sign of the degree of polarization is positive, which implies that the linear polarization develops in the direction parallel to the disk plane. The degree of linear polarization for observers with $\mu_{o b}=0.2$ and 0.5 show double peak structures.

$0.75<\mu_{S}<0.85$. The sign of the degree of polarization is positive, which implies that the polarization develops in the direction parallel to the disk plane. The lowest degree of polarization is obtained for an observer in the polar direction, which is similar to the case considered in Fig. 3.

It is very notable that the degree of linear polarization is not constant but shows symmetric double peak structure. This implies that the polarized flux shows quite different profiles from that of the total flux. The profile difference between the total flux and the polarized flux may be explained by the dependence of the relativistic Doppler effect on the line of sight. That is, the radiation in the polar direction suffers much less Doppler effect than the radiation in the equatorial direction. The highest degree of polarization and strongest polarized flux are obtained for an observer lying in the equatorial direction, because scattering occurs almost perpendicularly. Polarized fluxes have similar overall shape with the total profile for an observer with $\mu_{o b}=0.8$.

In Fig. 5, we show the same quantities from the scattering region in the equatorial direction $\mu_{S}=0.2$ as in Fig. 3 but having twice Thomson optical depth $\tau_{e}=0.8$. Because the scattering optical depth is large, 


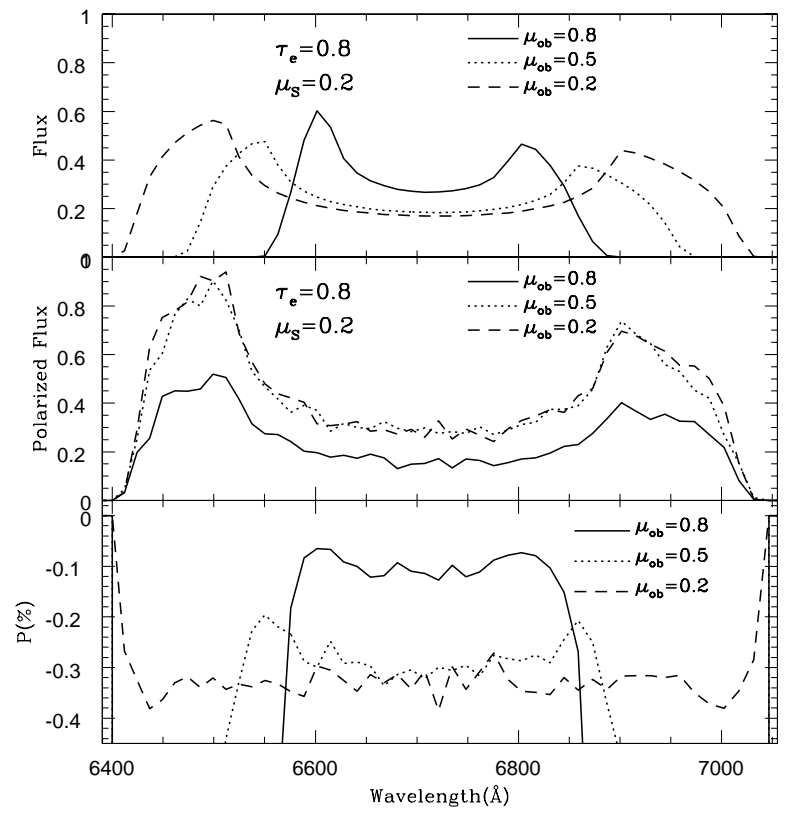

Fig. 5. - Same as Fig. 3 except that the Thomson optical depth of the scattering region is $\tau_{e}=0.8$. Linear degree of polarization and polarized flux are both stronger than those of Fig. 3.

more photons contribute to the total flux, enhancing the polarized flux and degree of linear polarization. In particular, the degree of linear polarization is larger than $0.3 \%$ for observers with $\mu_{o b}=0.2$ and $\mu_{o b}=0.5$. This may be contrasted with the case of $\tau_{e}=0.4$ where the degree of polarization is $0.24 \%$ for $\mu_{o b}=0.5$ and $0.26 \%$ for $\mu_{o b}=0.2$. A close examination also reveals that the total fluxes in Fig. 5 are slightly larger than the corresponding total fluxes in Fig. 3 due to increased contribution of scattered radiation in the larger Thomson optical depth condition.

In Fig. 6, we show the case of polar scattering region $\mu_{S}=0.8$ with Thomson scattering optical depth $\tau_{e}=0.8$. Again, the overall results are similar to those shown in Fig. 4 except stronger polarization due to the enhancement of scattered radiation. Interestingly the degree of linear polarization is nearly constant for an observer $\mu_{o b}=0.8$, but for other sightlines double peak structures are obtained. Under most favorable condition with $\mu_{o b}=0.2$ the degree of linear polarization may go up to $0.6 \%$, which is quite significant.

\section{SUMMARY AND DISCUSSION}

In this paper, we adopted a Monte Carlo method to compute the line profiles and polarization from a Keplerian disk around a Schwarzschild black hole. We considered two cases for the location of the scattering

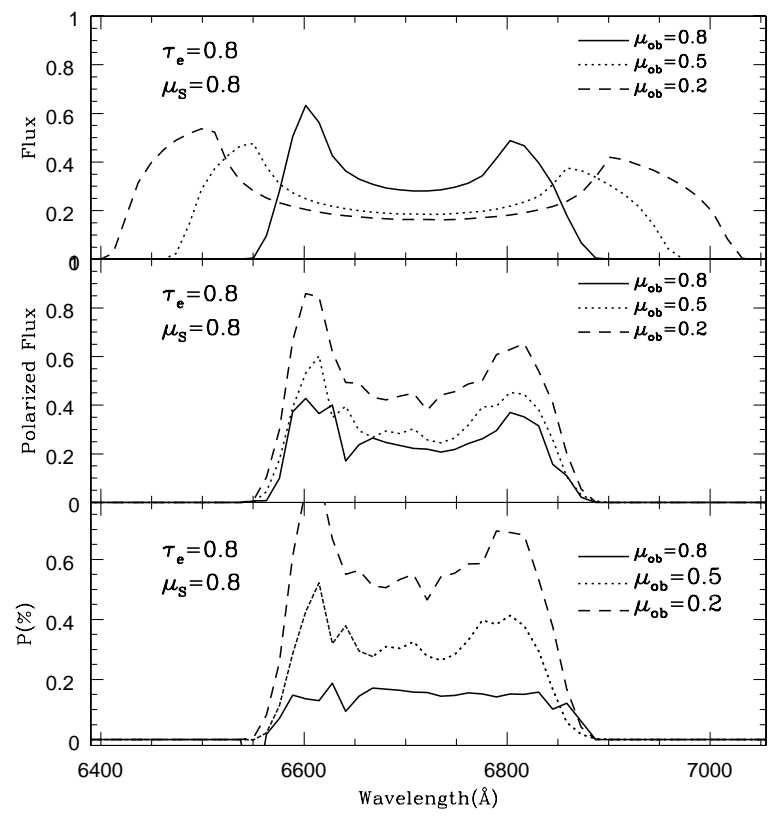

Fig. 6. - Same as Fig. 5 except that the scattering region lies in the polar direction with $0.75<\mu<0.85$. Note that in the lower panel, the degree of linear polarization is positive and therefore polarization develops in the direction parallel to the accretion disk.

region, that is, one lying in the equatorial direction and the other in the polar direction. The double peak structure persists in the total and polarized flux for all the observer's line of sight. However, the detailed shape and widths depend on the scattering geometry and observer's line of sight.

The width and overall shape of the polarized flux are mainly determined by the location of the scattering region relative to the emission region. For example, in the case where the scattering region is in the polar direction and the disk is seen almost edge-on, the line profile of the polarized flux is much narrower than that of the total flux. Therefore the separation of the peaks in the polarized flux may be used to determine the relative location of the free electron scattering region, whereas the smaller peak separation of the polarized flux indicates the scattering region in the direction normal to the accretion disk.

The degree of polarization is also affected by the Thomson scattering optical depth. Furthermore, the degree of linear polarization is not constant and therefore the profiles of the polarized flux differ from the total flux in a way characteristic of the scattering geometry. In this work, only axisymmetric scattering geometry is considered. If the scattering region is not azimuthally symmetric, then we may expect much more complicated structures both in the polarized flux and 
linear degree of polarization, which will be further complicated by the observer's line of sight. In this case, the position angle of polarization will be wavelengthdependent.

It is concluded that spectropolarimetry may shed much light on the geometry of the accretion disk in active galactic nuclei.

\section{ACKNOWLEDGMENTS}

The author is very grateful to an anonymous referee whose comments improved the presentation of the current paper. This work was supported by the faculty research fund of Sejong University in 2009.

\section{REFERENCES}

Antonucci, R. R. 1993, Unified Models for Active Galactic Nuclei and Quasars, ARA\&A, 31, 473

Antonucci, R. R., \& Miller, J. S. 1985, Spectropolarimetry and the Nature of NGC 1068, ApJ, 297, 621

Arav, N., Barlow, T. A., Laor, A., Sargent, W. L. W., \& Blandford, R. D. 1998, MNRAS, 297, 990

Blandford, R. D., \& McKee, C. F. 1982, Reverberation Mapping of the Emission Line Regions of Seyfert Galaxies and Quasars, ApJ, 255, 419

Chen, K., \& Halpern, J. P. 1989, Structure of LineEmitting Accretion Disks in Active Galactic Nuclei - Arp 102B, ApJ, 344, 115

Chen, K., Halpern, J. P., \& Filippenko, A. V. 1989, Kinematic Evidence for a Relativisitc Keplerian Disk: Arp 102B, ApJ, 339, 742

Eracleous, M., \& Halpern, J. P. 1994, Doubled-Peaked Emission Lines in Active Galactic Nuclei, ApJS, 90, 1

Eracleous, M., Livio, M., Halpern, J. P., \& StorchiBergmann, T. 1995, Elliptical Accretion Disks in Active Galactic Nuclei, ApJ, 438, 610

Gaskell, C. M. 1996, Evidence for Binary Orbital Motion of a Quasar Broad-Line Region, ApJ, 464, L107

Koratkar, A., Antonucci, R. R. J., Goodrich, R. W., Bushouse, H., \& Kinney, A. L. 1995, Quasar Lyman Edge Regions in Polarized Light, ApJ, 450, 501

Laor, A., Bahcall, J. N., Jannuzi, B. T., Schneider, D. P., Green, R. F., \& Hartig, G. F. 1994, The Ultraviolet Emission Properties of Five Low-Redshift Active Galactic Nuclei at High Signal-to-Noise Ratio and Spectral Resolution, ApJ, 420, 110

Laor, A. 2006, Evidence of Line Broadening by Electron Scattering in the Broad Line Region of NGC 4395, ApJ, 643, 112

Lee, H.-W., \& Ahn, S.-H. 1998, Polarization of the Ly alpha from an Anisotropically Expanding H I Shell in Primeval Galaxies, ApJ, 504, L61
Lee, H.-W., \& Blandford, R. D. 1997, On the Polarization of Resonantly Scattered Emission Lines - III. Polarization of Quasar Broad Emission Lines and Broad Absorption Line Troughs, MNRAS, 288, 19

Misner, C. W., Thorne, K. S., \& Wheeler, J. A. 1979, Gravitation, Freeman

Luminet, J. P. 1979, Image of a Spherical Black Hole with Thin Accretion Disk, A\&A, 75, 228

Osterbrock, D., \& Ferland, G. 2005, Astrophysics of Gaseous Nebulae and Active Galactic Nuclei, University Science Books

Puchnarewicz, E. M., Mason, K. O., \& Carrera, F. J. 1996, Double-Peaked Balmer Line Emission in the Radio-Quiet AGN RX J1042+1212, MNRAS, 283, 1311

Shapiro, S. L., \& Teukolsky, S. A. 1986, Black Holes, White Dwarfs and Neutron Stars: The Physics of Compact Objects, Wiley

Zheng, W., Sulentic, J. W., \& Binette, L. 1990, A Double-Stream Model for Line Profiles, ApJ, 365, 115 